\title{
High-J CO Intensity Measurements for Galaxies Observed by the Herschel FTS
}

\author{
Julia Kamenetzky ${ }^{1}$, Naseem Rangwala ${ }^{2}$, Jason Glenn ${ }^{3}$, \\ Philip Maloney ${ }^{3}$ and Alex Conley ${ }^{3}$ \\ ${ }^{1}$ Steward Observatory, University of Arizona, Tucson, AZ, U.S. \\ email: jkamenetzky@as.arizona.edu \\ ${ }^{2}$ NASA Ames Research Center, Mountain View, CA, U.S. \\ ${ }^{3}$ Center for Astrophysics and Space Astronomy, University of Colorado, Boulder, CO, U.S.
}

\begin{abstract}
Molecular gas is the raw material for star formation and is commonly traced by the carbon monoxide (CO) molecule. The atmosphere blocks all but the lowest-J transitions of CO for observatories on the ground, but the launch of the Herschel Space Observatory revealed the $\mathrm{CO}$ emission of nearby galaxies from $\mathrm{J}=4-3$ to $\mathrm{J}=13-12$. Herschel showed that mid- and high-J CO lines in nearby galaxies are emitted from warm gas, accounting for approximately $10 \%$ of the molecular mass, but the majority of the CO luminosity. The energy budget of this warm, highly-excited gas is a significant window into the feedback interactions among molecular gas, star formation, and galaxy evolution. Likely, mechanical heating is required to explain the excitation. Such gas has also been observed in star forming regions within our galaxy.

We have examined all $\sim 300$ spectra of galaxies from the Herschel Fourier Transform Spectrometer and measured line fluxes or upper limits for the $\mathrm{CO} \mathrm{J}=4-3$ to $\mathrm{J}=13-12$, [CI], and [NII] 205 micron lines in $\sim 200$ galaxies, taking systematic effects of the FTS into account. We will present our line fitting method, illustrate trends available so far in this large sample, and preview the full 2-component radiative transfer likelihood modeling of the $\mathrm{CO}$ emission using an illustrative sample of 20 galaxies, including comparisons to well-resolved galactic regions. This work is a comprehensive study of mid- and high-J CO emission among a variety of galaxy types, and can be used as a resource for future (sub)millimeter studies of galaxies with ground-based instruments.
\end{abstract}

Keywords. galaxies: ISM - ISM: molecules - submillimeter

\section{Introduction}

Molecular gas is the raw material for star formation, and it is also influenced by the ongoing processes of star formation, life, and death. This work focuses on CO as a tracer of molecular gas, specifically the new view of the high-J lines (up to $\mathrm{J}=13-12$ ) afforded to us by the Herschel Fourier Transform Spectrometer. The atmosphere blocks all but the lowest-J transitions of CO for observatories on the ground, but the launch of the Herschel Space Observatory revealed the CO emission of nearby galaxies from $\mathrm{J}=4-3$ to $\mathrm{J}=13-12$.

The early science results from the Herschel demonstrated that the high-J emission from $\mathrm{CO}$ was significantly higher than predicted by standard, $T_{k i n}<100 \mathrm{~K}$ molecular gas described by the low-J lines in the nearby starburst galaxy M82 (Panuzzo et al. 2010). Subsequent studies, such as Kamenetzky et al. (2014) and references therein, found this was true for a variety of galaxies, shown in Figure 1. 


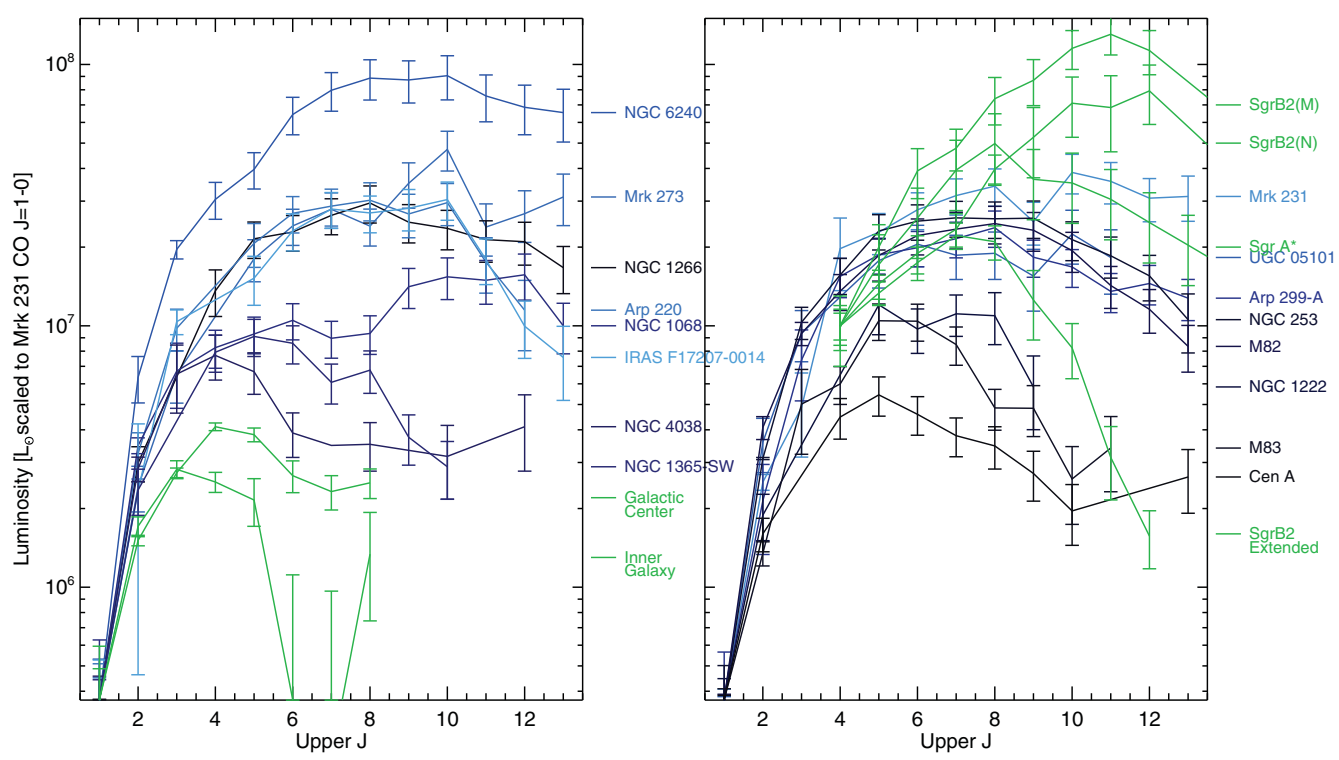

Figure 1. Normalized CO SLEDS, figure and caption reproduced from Kamenetzky et al. (2014) All $J=1-0$ luminosities are scaled to match that of Mrk $231\left(3.7 \times 10^{5} \mathrm{~L}_{\odot}\right)$. SLEDs are colored to indicate increasing $L_{\mathrm{FIR}}$ with increasing lightness. Placement in the left or right panel is for clarity only. On the left panel, the SLEDs of the Galactic center $(|l|<2.5)$ and the Inner Galaxy $\left(2.5<|l|<32^{\circ} .5\right)$, also normalized, are shown in green for comparison (Fixsen et al. 1999). On the right panel, we show the SLEDs of two star-forming cores and the extended envelope of Sgr B2 (Etxaluze et al. 2013) and that of Sgr A* (Goicoechea et al. 2013). Because these SLEDs begin at $J=4-3$, we scale $J=4-3$ to $10^{7} \mathrm{~L}_{\odot}$ for visual comparison of the shapes. None of the SLEDs are corrected for dust extinction.

\section{New Survey}

This new work is a compilation of all extragalactic SPIRE FTS spectra from the Herschel mission. These proceedings summarize the findings of Kamenetzky et al. 2015, submitted to The Astrophysical Journal. To briefly summarize the processing methodology for all $\sim 300$ spectra from the archive, we first corrected for source/beam coupling using the SPIRE PSW photometry maps. We fit the CO $J=4-3$ through $J=13-12$ lines, two [CI] lines, and [NII] $205 \mu \mathrm{m}$ line using an approach which better takes into account the systematic, line-profile-like ringing of the SPIRE spectrum better than least squares minimization. Additional low-J $(J=1-0, J=2-1, J=3-2)$ lines were collected from the literature and obtained at the the Arizona Radio Observatory.

\section{Findings}

As shown in Figure 2, we find a superlinear slope in CO $J=1-0$ for the relation $\log \left(L_{\mathrm{FIR}}\right)=\mathrm{a} \log \left(L_{\mathrm{CO}}^{\prime}\right)+\mathrm{b}$. As one moves to higher-J lines, the relation flattens and stays linear by about $J=5-4$. Individual populations (e.g. only (U)LIRGS) show some variation in the line fits.

The consequence of the aforementioned linear fits is that the ratio of high-J to low-J CO line luminosities only varies by about a factor of 10 throughout this whole sample (Figure 3), which covers many orders of magnitude of far infrared luminosity (and star formation rate). We found that many models predict steep drop-offs in high-J to low-J ratios with lower star formation rates, such as Narayanan \& Krumholz (2014). The models 


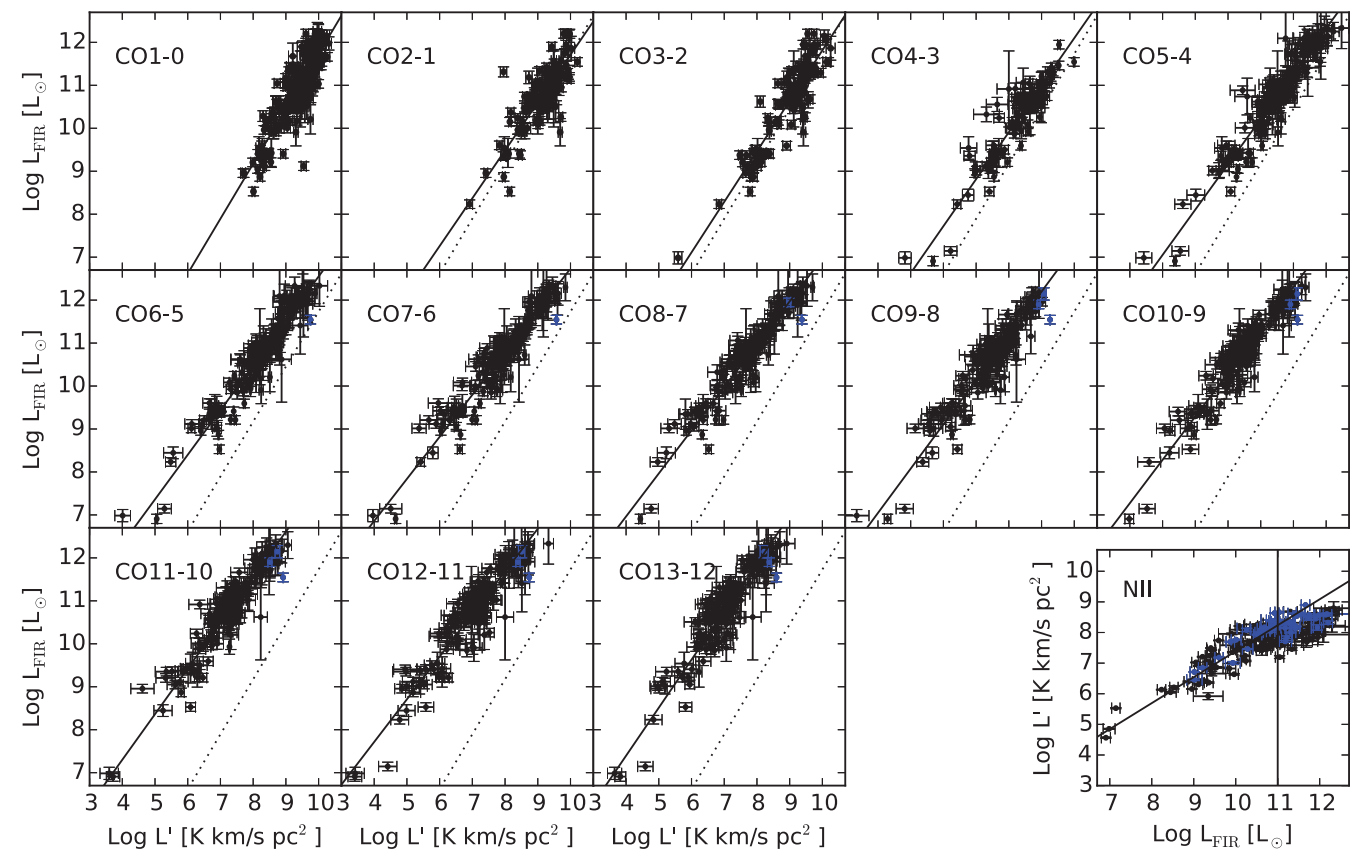

Figure 2. CO vs. $L_{\mathrm{FIR}}$, figure and caption reproduced from Kamenetzky et al. (2015, submitted). The y-axis is the $L_{\mathrm{FIR}}$ in the beam for comparison to the CO measurement. Low- $J$ lines may include multiple measurements for the same galaxy if available in the literature. Blue data points indicate resolved (Sinc-Gaussian) measurements from the FTS. The CO $J=1-0$ line fit is shown as a dotted line on each other CO plot for comparison. The [NII] line is shown with the $\mathrm{x}$ - and y-axes flipped compared to $\mathrm{CO}$ as is more common. It is only fit up to $\log \left(L_{\mathrm{FIR}}\right)=$ 11 due to the well-known line deficit above this value.

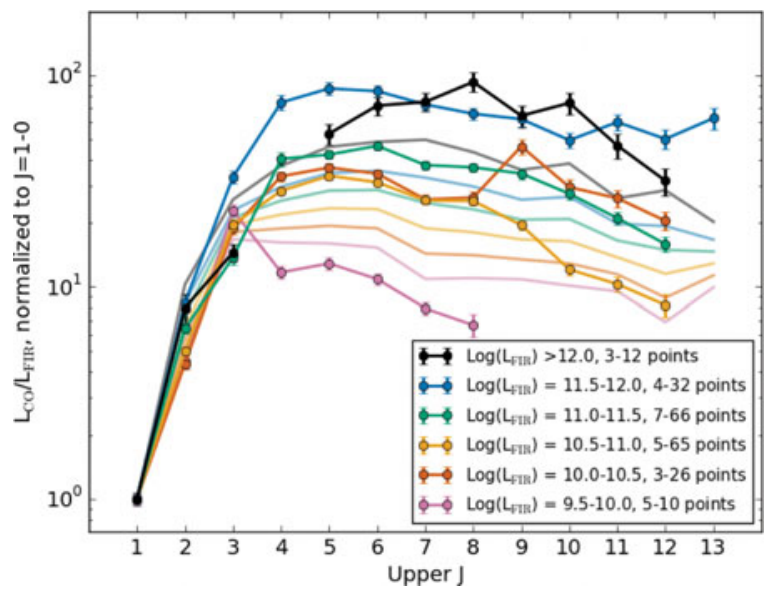

Figure 3. Average SLEDs by $L_{\mathrm{FIR}}$ Ranges, figure and caption reproduced from Kamenetzky et al. (2015, submitted). For $L_{\mathrm{FIR}}$ bin ranges shown in the legend, the value of $L_{\mathrm{CO}} / L_{\mathrm{FIR}}$ was averaged if measurements existed for at least 3 galaxies. All SLEDs were then divided by the value of the $\mathrm{CO} J=1-0$ line to demonstrate the difference in relative excitation of the $\mathrm{CO}$ ladder. The number of data points used in each SLED may change with each line, which is why a range is given in the legend. The highest luminosity bin is dominated by more distant galaxies, where the CO $J=4-3$ line is likely to be redshifted out of the FTS band, which is why that black data point is missing. The lighter lines (in both plots) with no data markers indicate the predictions from the slopes in Figure 2 for the center of each log bin. 
of Kazandjian et al. (2015) include mechanical heating, which can produce flatter SLEDs, but only at much higher ratios than we see here.

\section{Conclusions}

This catalog of $\mathrm{CO},[\mathrm{CI}]$, and $[\mathrm{NII}]$ lines is created using a uniform processing method for dealing with source/beam coupling and proper estimates of the line flux measurements, given the correlated nature of spectral bins in the SPIRE FTS. Over multiple orders of magnitude, the mid- to high-J CO lines show linear slopes for the relation $\log \left(L_{\mathrm{FIR}}\right)=\mathrm{a} \log \left(L_{\mathrm{CO}}^{\prime}\right)+\mathrm{b}$. As a result, the average SLEDs show increasing mid- to high-J CO luminosity with increasing $L_{\mathrm{FIR}}$, but only in the range from a few to $\sim 100$. Current theoretical models do not reproduce flat SLEDs with appropriate ratios. More details are available in Kamenetzky et al. (2015, submitted).

\section{References}

Etxaluze, M. et al. 2013, Astron. Astrophys., 556, A137

Fixsen, D. J., Bennett, C. L., \& Mather, J. C. 1999, Astrophys. J., 526, 207

Goicoechea, J. R. et al. 2013, Astrophys. J., 769, L13

Kamenetzky, J., Rangwala, N., Glenn, J., Maloney, P. R., \& Conley, A. 2014, Astrophys. J., 795,174

Kamenetzky, J., Rangwala, N., Glenn, J., Maloney, P. R., \& Conley, A. 2015, submitted to Astrophys. J., arXiv:1508.05102

Kazandjian, M. V., Meijerink, R., Pelupessy, I., Israel, F. P., \& Spaans, M. 2015, Astron. Astrophys., 574, A127

Narayanan, D. \& Krumholz, M. R. 2014, Mon. Not. R. Astron. Soc, 442, 1411

Panuzzo, P. et al. 2010, Astron. Astrophys., 518, L37 